\title{
A new technique of ultrasound guided percutaneous renal biopsy by perforated probe and perpendicular needle trajectory
}

\author{
Simone Brardi ${ }^{1}$, Gabriele Cevenini ${ }^{2}$, Angelo Giovanni Bonadio ${ }^{3}$ \\ ${ }^{1}$ Hemodialysis Unit, S. Donato Hospital, Arezzo, Italy; \\ 2 Department of Medical Biotechnologies, University of Siena, Italy; \\ ${ }^{3}$ Pathological Anatomy 1, University of Pisa, Italy.
}

\begin{abstract}
Summary The percutaneous biopsy of native kidneys according to the classical methodology is performed under real time ultrasound guidance with the needle introduction along a trajectory of about $30^{\circ}$, aimed to the lower pole of the kidney. Recently, a variant of the classical technique has been introduced by which a perforated ultrasound probe is used to guide the needle along a perpendicular trajectory to the terminal section of the lower kidney pole where the front and back margins of the cortical kidney tissue join each other without renal sinus interposition so to offer to the needle a 3-4 cm thick cortical tissue front which allows to obtain a cortical tissue sample suitable for histological examination even with a single needle pass, while at the same time limiting the possibility of damaging the smaller kidney calices of the lower group whose lesion causes hematuria.

In this paper, we present a large survey (50 patients) to compare to data from the literature obtained by using similar needle gauge and with a similar follow-up period after biopsy.

The result of this comparison confirms the efficacy of this variant of the classical technique because in front of a statistically lower number of needle passes, it allowed to obtain 100\% of samples suitable for histological analysis, in absence of major complications and with a statistically lower post-biopsy hemoglobin drop in comparison to that observed in a group of 44 patients biopsied with a greater number of needle passes, in the only study of the literature which is directly comparable to our study in relation to needle gauge and duration of monitoring.
\end{abstract}

KEY WORDS: Percutaneous renal biopsy; Perforated ultrasound probe; Perpendicular needle trajectory; Number of needle passes.

Submitted 30 October 2017; Accepted 15 November 2017

\section{INTRODUCTION}

Percutaneous kidney biopsy is an important and often irreplaceable instrument for the diagnosis and treatment of kidney disease although it may be associated with multiple complications, most commonly with bleeding which in rare cases can lead to sometimes fatal retroperitoneal bleeding (1-3).

For this reason, the renal biopsy technique has evolved over the years to increase its safety until the introduction of spring-loaded needle biopsy device and of real-time ultrasound guidance which are currently considered the "gold standard" procedure for the percutaneous renal biopsy as they allow to minimize the incidence of associated complications (3).

According to the classical methodology, as proposed by the guidelines, with the patient in the prone position, the biopsy needle is driven according to a sagittal ultrasound scan, with an angle of about 30 degrees towards the lower kidney pole. Usually two needle passes are carried in order to obtain a sufficient amount of renal parenchyma tissue for optic microscopy, immunofluorescence and electron microscopy analysis when necessary (1).

Recently, in a limited series (4), a variant of the classical technique has been proposed according which placing the patient in the prone position the biopsy needle is driven by a perforated ultrasound probe with a perpendicular trajectory in order to reach the more lower portion of the lower kidney pole, where the front and back margins of the renal cortical join each other without renal sinus interposition (Figure 1). By the use of the perforated probe, the needle advances perpendicularly toward a $3-4 \mathrm{~cm}$ thick cortical tissue front, without the renal sinus interposition, allowing to collect a suitable sample of cortical kidney tissue with a single needle pass and, at the same time, limiting the possibility of damage to the smaller kidney calices of the lower group whose lesion causes hematuria (5).

The first purpose of this technique is therefore to obtain with only one needle pass (compared to the two or more required according to the classical method) (1) and thus with less kidney parenchyma trauma, a quantity of material sufficient to put an histological diagnosis and at the same time to limit the possibility of damaging the calices

\section{Figure 1.}

The path of the needle according to the technique of percutaneous renal biopsy with perforated probe and perpendicular needle trajectory.

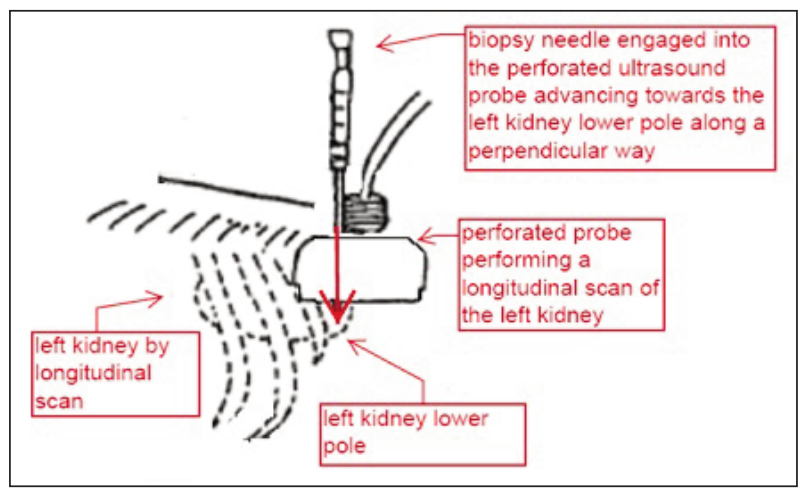

No conflict of interest declared. 
present in the renal sinus and therefore the possibility of bleeding because the biopsy is performed where the front and back margins of the cortical kidney tissue join each other without the renal sinus interposition.

In the following paper we present therefore a survey carried out in a large population with this technique in order to confirm or not the supposed advantages offered by this technique.

\section{SubJeCtS AND METHOdS}

Compared to the previously published pilot survey that counted only 26 cases (4), for this paper.

We retrospectively evaluated 50 consecutive patients submitted to percutaneous renal biopsy by the technique of the perforated ultrasound probe with perpendicular needle trajectory at the Nephrology ward of the San Donato Hospital of Arezzo from November 2012 to October 2017. The mean age of the 50 patients (32 males and 18 females) was $52.7 \pm 16.7$ (mean $\pm \mathrm{SD}$, range 18-83) years, with average creatinine values (before the biopsy) of $1.7 \pm 1.7 \mathrm{mg} / \mathrm{dl}$. (Table 1 ).

The bleeding time, prothrombin time, and platelet counts were within the normal range across all patients. Each patient, before the procedure, was subjected to renal ultrasound in order to exclude the presence of any of the known contraindications for the execution of renal biopsy (5) and any platelet anti-aggregant and/or anticoagulant was stopped 7 days before the procedure (6).

In the case of arterial hypertension, a sufficient control of arterial pressure values was assured, eventually with the addition to the therapy of a calcium channel blocker. Finally an informed consent was obtained from each patient (7). Percutaneous kidney biopsies were always performed on native kidneys and always at the lower left kidney pole with the exception of two cases (with solitary kidneys) where the biopsy was performed at the lower right kidney pole.

For real-time ultrasound guidance, a Hitachi convex 3.5hhz perforated ultrasound probe coupled to a Hitachi Astro 256 ultrasonograph was used.

In all cases, the Bard monopty needles (Bard biopsy systems, Tempe, Arizona, USA) were used with needle gauge size and length of 16 Gauge x $16 \mathrm{~mm}$.

Renal biopsy was performed with the patient in the prone position while a pillow was placed under the abdomen of the patient (5). After disinfection of the skin with povidone iodine and local anesthesia (lidocaine $20 \mathrm{mg} / \mathrm{ml}$ ) under real-time ultrasound guidance, according to longitudinal scan planes on the rear axillary line and tilting the probe towards the spine, the needle was directed to the more

\section{Table 1.}

Characteristics of the patients enrolled.

\begin{tabular}{|lc|}
\hline Patients, $\mathbf{n}$. & 50 \\
\hline Age (years) & $52.72 \pm 16.70$ \\
\hline Range (years) & $18-83$ \\
\hline Male/female, $\mathbf{n}$. & $32 / 18$ \\
\hline Pre-biopsy creatinine (mg/dl) & $1.66 \pm 1.68$ \\
\hline${ }^{*}$ mean \pm SD & \\
\hline
\end{tabular}

\section{Figure 2.}

The moment of needle drive operation with perpendicular needle trajectory; to note the absence of renal sinus interposed.

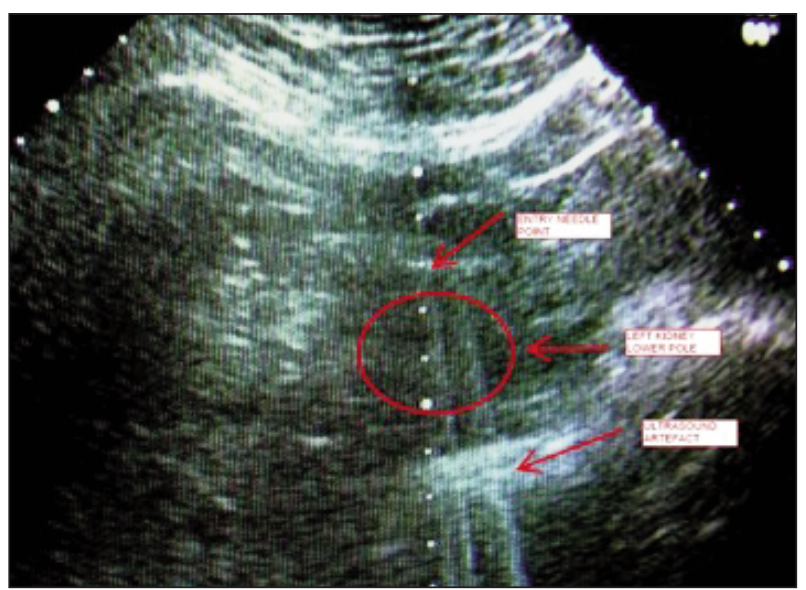

lower portion of the kidney lower pole where the front and back margins of the cortical kidney tissue join each other without the renal sinus interposition, along a bound path perpendicular to the lower pole by the needle passage of a special adapter for the zero degrees angle previously inserted on the ultrasound perforated probe (Figure 2).

Only one needle pass was carried out; the needle pass was repeated only when the sample was not suitable to a first optical analysis. In particular insufficient sampling happened only in those patients who, at the moment of the firing of the needle mechanism for the core sampling, moved for fear or inability to keep the position set (4).

All the patients were then subjected to ultrasound imaging immediately after the biopsy, at a distance of about 6 hours and ultimately after the first 24 hours $(1,4)$.

Besides, all the patient, after the biopsy, remained in bed for the first 24 hours as recommended by the Italian national guidelines $(1,4)$.

Before the procedure a blood sample was drawn for blood count that was repeated six hours after the biopsy, while subsequent blood count collections were dictated by the possible presence of hematomas or by major reductions of the hemoglobin at the six-hour control after the biopsy $(1,4)$.

Urination was monitored to exclude macrohaematuria and the blood pressure was measured intensively for the first two hours and then at regular intervals of eight hours $(1,4)$.

\section{RESULTS}

Even in the larger survey that we present here, as in the preliminary data (4), the biopsy sampling (examined for the most at the Pathological Anatomy 1 of the University of Pisa) was successful (more than 5 glomeruli per biopsy specimen and/or a validation by the anatomopathologist about the presence of sufficient material to diagnose) $(5,8)$ in the $100 \%$ of the cases. The sample then was obtained at the first and only needle pass in $90 \%$ of cases (with a slight increase compared to the $88.5 \%$ rate found in the first published survey) (4). 
As in the pilot casuistry, no major complications of renal biopsy were observed and in particular transient macrohaematuria was found only in a patient and no patient was subjected to blood transfusion or death (Table 2). However, we must point out that a female patient was transfused far beyond 24 hours of observation due to the failure to observe the expected period of bed rest, so it was necessary a further bed forced rest period but no embolization was required.

However subcapsular hematomas was found in $40 \%$ of the patients (with a slight reduction versus the 42\% found in the pilot survey) but all hematomas were of a non-significant type (i.e. with a maximum thickness of $\leq 2 \mathrm{~cm}$ as by definition of Italian guidelines) (1) (Table 2). Finally, the mean hemoglobin value was reduced from the pre-biopsy value of $12.4 \pm 2.1 \mathrm{~g} / \mathrm{dl}$ to the post-biopsy value of $12.0 \pm 2.03 \mathrm{~g} / \mathrm{dl}$. However, a decrease in hemoglobin values $\geq 2 \mathrm{~g} / \mathrm{dl}$ (considered significant or associated with increased risk of complications) (9) was reported only in $4.25 \%$ of patients (Table 2).

\section{Table 2.}

Results.

\begin{tabular}{|lc|}
\hline Patients, $\mathbf{n}$. & 50 \\
\hline Needle passes, $\mathbf{n}{ }^{*}$ & $1.10 \pm 0.30$ \\
\hline Sample adequacy for the histological analysis & $100 \%$ \\
\hline Small non-significant hematomas, $\mathbf{n}$. & $20(40 \%)$ \\
\hline Large hematomas, $\mathbf{n}$. & 0 \\
\hline Macrohematuria, $\mathbf{n}$. & 1 \\
\hline Embolizations, $\mathbf{n}$. & 0 \\
\hline Blood transfusions, $\mathbf{n}$. & 0 \\
\hline Death, $\mathbf{n}$. & 0 \\
\hline Hemoglobin decrease (g/dl) to $\mathbf{6}$ hours after biopsy & $-0.44 \pm 0.89$ \\
\hline $\begin{array}{l}\text { Hemoglobin fall } \geq \mathbf{2} \text { gr/dl after biopsy as percent } \\
\text { of the total patients }\end{array}$ & $4.25 \%$ \\
\hline * mean \pm SD & \\
\hline
\end{tabular}

\section{Discussion}

Since the present series did not include controls, as our preliminary study previously published, in order to assess effectiveness and safety of this variant of the classical biopsy technique we had to make a comparison with literature data homogeneous for needle gauge and length of post-bioptic monitoring period (4).

For this comparison homogeneity with regard to needle gauge is important as 18 Gauge needles were found to have a greater number of complications (10) (probably due to the greater number of needle passes necessary to obtain adequate material for diagnosis) (11) and fewer biopsy specimens in comparison to the use of 14 or 16 Gauge needles (10). Furthermore in the meta-analysis of Corapi et al. (12) there was an increase in the number of blood transfusions in studies with 14 Gauge needles compared to 16 or 18 Gauge needles.

Equally important for comparison is the homogeneity of the patient's monitoring period after kidney biopsy, which must be at least 12 hours and better over 24 hours (1), since $33 \%$ of the complications occur after the first eight hours and $91 \%$ within 24 hours $(1,13)$.

So we have selected within the largest meta-analysis at the time available in literature, that is Corapi et al. (12), the studies comparable to our study for needle gauge and duration of the monitoring with sufficient data to calculate the confidence interval for the number of needle passes per patient and the variations of hemoglobin between the pre and post-biopsy value at a distance of about six hours. We identified three papers from which, for completeness of data, we have been able to extrapolate the $95 \%$ confidence interval for the number of needle passes carried out, namely Maya and Allon's study of 2009 (6), for which it is necessary underline that both needles 16 and 18 Gauge were used, Lin et al. study of 2006 (14), where 16 Gauge needles were used for adult patients and 18 Gauge needles for pediatric patients (which number is not specified) and, finally, Ori et al. study of 2002 (15) where only 16 Gauge needles were used.

In all three studies selected for comparison, a histological sample sufficient for diagnosis was obtained for almost all patients, though with a number of needle passes greater than in our study.

In Maya and Allon's paper (6) with a 100\% validity of the sample for the histological analysis, $1.6 \pm 0.8$ needle passages (IC 95\%: 1.44-1.76) were performed in the 100 patients enrolled.

In the paper of Lin et al. (14) with a 100\% validity of the sample for the histological analysis, $2.2 \pm 0.6$ passages were performed in 183 outpatients (IC 95\%: 2.11-2.29) and $2.3 \pm 0.7$ passages were performed in 147 inpatients (IC 95\%: 2.19-2.41).

In the paper of Ori et al. (15) in 44 biopsied patients with an older model of ultrasonograph with a $98 \%$ of sample adequacy for histological analysis, $4.7 \pm 0.3$ passages (IC 95\%: 4.61-4.79) were performed, while in 41 biopsied patients with a newer ultrasonograph, with a $95 \%$ of sample adequacy for histological analysis, $4 \pm 0.1$ passages (IC 95\%: 3.97-4.03) were performed, that is a statistically significant smaller number of needle passes when compared to the other group of 44 patients (Table 3 ).

By comparing these data with those of our larger survey in which with a $100 \%$ of sample adequacy for the histological analysis, $1.1 \pm 0.30$ needle passes (IC 95\%: 1.021.18) were performed, it clearly emerges a statistically significant difference already found in our preliminary series (4) (Table 3).

Regarding the complications related to renal biopsy and specifically the hemoglobin variations between the prebiopsy value and the one found after an average interval of 6 hours from the biopsy, we could only compare Maya and Allon's paper (6) and the paper of Ori et al. (15) since in the work of Lin et al. (14) blood count was not routinely collected in all patients after renal biopsy.

Then comparing 95\% confidence intervals for the pre and post-biopsy variation of hemoglobin, we observed that there was no statistically significant difference with Maya and Allon work data (as in the pilot survey already published) (6), while there was a statistically significant difference with the subgroup of 44 patients undergoing biopsy with the aid of an older ultrasonograph in the work of Ori et al. (15) where the hemoglobin reduction was significantly greater than in our patients.

Indeed there was no statistically significant difference between our data and those of the second group of 41 
Table 3.

95\% confidence interval (Cl) for the needle passes.

\begin{tabular}{|c|c|c|c|c|c|}
\hline $\begin{array}{l}\text { Author } \\
\text { (years of publication) }\end{array}$ & Patients, n. & Needle gauge & $\begin{array}{c}\text { Number } \\
\text { of needle passes } \\
\text { (mean } \pm \text { SD) }\end{array}$ & $95 \% \mathrm{Cl}$ & $\begin{array}{c}\text { Duration of } \\
\text { post-biopsy monitoring } \\
\text { (hours) }\end{array}$ \\
\hline Maya and Allon (2009) & 100 & $\begin{array}{l}16 \text { e } 18 \text { Gauge } \\
\text { (but the } 16 \text { Gauge was } \\
\text { used more frequently) }\end{array}$ & $1.6 \pm 0.8$ & $1.44-1.76$ & 8 \\
\hline \multirow[t]{2}{*}{ Lin et al. (2006) } & 183 (outpatients) & $\begin{array}{l}16 \text { and } 18 \text { Gauge } \\
\text { (but the } 16 \text { Gauge was } \\
\text { used more frequently) }\end{array}$ & $2.2 \pm 0.6$ & $2.11-2.29$ & 6 \\
\hline & 147 (inpatients) & & $2.3 \pm 0.7$ & $2.19-2.41$ & \\
\hline \multirow[t]{2}{*}{ Ori et al. (2002) } & $\begin{array}{c}44 \text { (subject to biopsy } \\
\text { by an older } \\
\text { ultrasonograph) }\end{array}$ & 16 & $4.7 \pm 0.3$ & $4.61-4.79$ & 24 \\
\hline & $\begin{array}{c}41 \text { (subjected to biopsy } \\
\text { by a newer } \\
\text { ultrasonograph) }\end{array}$ & 16 & $4 \pm 0.1$ & $3.97-4.03$ & \\
\hline Brardi S et al. (2017) & 50 & 16 & $1.10 \pm 0.30$ & $1.02-1.18$ & 24 \\
\hline
\end{tabular}

patients in the same work by Ori et al. (15) that were biopsied with the aid of a newer ultrasonography that, as already mentioned, were subjected to a statistically minor number of needle passes than the other group in the same study (Table 4).

However, to properly evaluate the outcome of this comparison it is necessary to keep in mind that while in the work of Ori et al. (15), as in ours, 16 Gauge needles were used and the patient remained in bed for the first 24 hours, 18-Gauge needles were used in Maya's and Allon's work (6), and the observation time was limited to only eight hours.

Since, as well explained in Whittier et al. (13), the clinical recognition of major complications increases proportionally to observation time, it is legitimately possible to assume that an observation time of only eight hours (as in the Maya and Allon's work) (6) may have lost up to $33 \%$ of later post-biopsy complications and this is the reason why the only work that can be compared with ours (for the needle gauge and for the duration of the monitoring period) is that of Ori et al. (15).

Likewise, and for the same reasons, regarding to the major complications of renal biopsy (macrohematuria, blood transfusions or deaths) and the formation of post-biopsy hematomas, the only work comparable with ours remains that of Ori et al. (15) where in front of only one patient with gross hematuria, two patients ( $4.5 \%$ of the total) in

Table 4.

95\% confidence interval (Cl) for the hemoglobin variations after biopsy.

\begin{tabular}{|c|c|c|c|}
\hline $\begin{array}{l}\text { Author } \\
\text { (years of publication) }\end{array}$ & Patients, n. & $\begin{array}{l}\text { Hemoglobin } \\
\text { variations } \\
(\mathrm{g} / \mathrm{dl})^{*}\end{array}$ & $\begin{array}{c}95 \% \mathrm{Cl} \text { for the } \\
\text { hemoglobin variations } \\
(\mathrm{g} / \mathrm{dl})^{*}\end{array}$ \\
\hline Maya and Allon (2009) & 100 & $-0.54 \pm 0.29$ & $-0.48-0.6$ \\
\hline \multirow[t]{2}{*}{ Ori et al. (2002) } & $\begin{array}{c}44 \text { (subjected to } \\
\text { biopsy by an older } \\
\text { ultrasonograph) }\end{array}$ & $-0.86 \pm 0.16$ & $-0.91-0.81$ \\
\hline & $\begin{array}{c}41 \text { (subjected to } \\
\text { biopsy by a newer } \\
\text { ultrasonograph) }\end{array}$ & $-0.47 \pm 0.10$ & $-0.5-0.44$ \\
\hline Brardi S et al. (2017) & 50 & $-0.44 \pm 0.89$ & $-0.18-0.69$ \\
\hline
\end{tabular}

the group of 44 patients subjected to biopsy with an older ultrasonograph and two patients $(4,9 \%$ of the total) of the group of 41 biopsied patients with a newer ultrasonograph were transfused, while in our present larger survey in front of only one patient with gross hematuria no major complication was reported and no patient was transfused except one female that did not comply with post-biopsy bed resting and had to be transfused after 24 hours.

In the same work by Ori et al. (15) significant hematomas were also reported in 5 patients (equal to $11.4 \%$ of the total) of the group of 44 patients subject to biopsy with an older ultrasonograph and in 2 patients (equal to $4.9 \%$ of the total) of the group of 41 patients subjected to biopsy with a newer ultrasonograph, while in our larger survey, as well as in the preliminary study (4), only non-significant (i.e. with a maximum thickness of $\leq 2 \mathrm{~cm})(1,2)$ hematomas were found in 20 patients equal to $40 \%$ of the total (Table 2 ). This high number of non-significant hematomas, however, may be related to the fact that while in the work of Ori et al. (15) after ultrasound examination performed at the time of biopsy, no further ultrasound tests were performed except in the case of complications, in our protocol, renal ultrasound was repeated at 6 and 24 hours after the biopsy in all patients regardless of any symptomatology.

\section{Conclusions}

The data provided by the present larger survey confirm the findings of our preliminary series (4) though the lack of a control group is still a limitation of the strength of our conclusions. On this basis we believe that the percutaneous renal biopsy with perforated ultrasound probe and perpendicular needle trajectory, performed in that portion of kidney lower pole where the front and back margins of the cortical kidney tissue join each other without the renal sinus interposition, allows to obtain (in native adult kidneys) samples suitable for histological analysis comparable to those provided by repeated needle passes performed according to the classical technique. The statistically lower number of passages is explained by the abundance of cortical tissue that the needle crosses.

Performing the needle pass in that portion of the lower pole of the kidney where there is no renal sinus interposition and so avoiding to damage the smaller kidney calices of the lower group whose lesion causes hematuria (5) achieved in this larger survey as in the preliminary smaller sample (4), a post-biopsy reduction of the hemoglobin statistically lower than that observed in a subgroup of 44 biopsied patients with an older ultrasonograph and therefore subjected to a statistically significant greater number of needle passes Ori et al. (15). 
As already pointed out in the study of Eiro et al. (7) and also reported by Zhu et al. (16) in a recent review of factors that can minimize post-biopsy bleeding, although the frequency of needle passes does not differ significantly between cases with moderate complications and cases with no or only mild complications, the frequency of needle passes however represents an independent risk factor, as evidenced by the multivariate logistics analysis conducted in the work of Eiro et al. (7).

Besides the frequency of the needle passes is amongst all (age $>60$ years, marked hypertension with PAS $>140$ or $\mathrm{PAD}>90 \mathrm{mmHg}$ and amyloidosis) the only risk factor for bleeding, as highlighted in the work of Eiro et al. (7), that can be addressed using a technique such as described here, oriented to minimize the number of the needle passes.

To confirm this we can also remember the already mentioned fact that with the use of 18 Gauge needles, despite a lower caliber, a greater number of complications were found, probably due to the greater number of needle passes required to obtain valid samples for the histological analysis $(10,11)$.

\section{REFERENCES}

1. Commissione SIN-SIAPEC. Requisiti per la biopsia renale: diagnostica nefropatologica ed esecuzione clinica. Release 1. In: Best practice; www.nephromeet.com. 12 Novembre 2015.

2. Parrish AE. Complications of percutaneous renale biopsy: a review of 37 years'experience. Clin Nephrol. 1992; 38:135-41.

3. Brachemi S, Bollèe G. Renal biopsy practice: what is the gold standard? World J Nephrol. 2014; 3:287-294.

4. Brardi S, et al. Una variante della tecnica classica eco guidata della biopsia renale percutanea: l'approccio perpendicolare in scansione longitudinale con sonda forata. GIN. 2017; 34:146-156.

5. Meola M, Barsotti G, Cupisti A, et al. Free-Hand ultrasound- guided renal biopsy: report of 650 consecutive cases. Nephron. 1994; 67:425-430.

6. Maya ID, Allon M. Percutaneous renal biopsy: outpatient observation without hospitalization is safe. Seminars in Dialysis. 2009; 22:458-461.

7. Eiro M, Katoh T, Watanabe T. Risk factors for bleeding complications in percutaneous renal biopsy. Clin Exp Nephrol. 2005; 9:40-45.

8. Geldenhuys L, Nicholson P, Sinha N, et al. Percutaneous native renale biopsy adequacy: a successful interdepartmental quality improvement activity. Canadian Journal of Kidney Health and Disease 2015; 2:8.

9. Visconti L, Cernaro V, Ricciardi CA, et al. Renal biopsy: still a Landmark for the nephrologist. World J Nephrol. 2016; 5:321-327.

10. Tøndel C, Vikse BE, Bostad L, Svarstad E. Safety and complications of percutaneous kidney biopsies in 715 children and 8573 adults in Norway 1988-2010. Clin J Am Soc Nephrol. 2012; 7:1591.

11. Cagnoli L, Fuiano G, Imbasciati E, et al. Linee Guida sulle indicazioni ed esecuzione della biopsia renale percutanea e sulla terapia delle nefropatie glomerulari. Linee Guida SIN Revisione 2003. Giornale Italiano di Nefrologia. 2003; 20:S3-S47.

12. Corapi KM, Chen JL, Balk EM, Gordon CE. Bleeding complications of native kidney biopsy: a systematic review and meta-analysis. Am J Kidney Dis. 2012; 60:62.

13. Whittier W L, Korbet S M. Timing of complications in percutaneous renal biopsy. J Am Soc Nephrol. 2004; 15:142-147.

14. Lin WC, Yang Y, Wen YK, Chang CC. Outpatient versus inpatient renal biopsy: a retrospective study. Clinical Nephrology. 2006; $66: 17-24$.

15. Ori Y, Neuman H, Chagnac A, et al. Using the automated biopsy gun with real-time ultrasound for native renale biopsy. IMAJ. 222; 4:698-701.

16. Zhu MS, Chen JZ, Xu AP. Factors that can minimize bleeding complications after renale biopsy. Int Urol Nephrol. 2014; 46:19691975.

\section{Correspondence}

Simone Brardi, MD

sibrardi@gmail.com

Hemodialysis Unit, S. Donato Hospital, Arezzo, Italy

Gabriele Cevenini, MD

Department of Medical Biotechnologies, University of Siena, Italy

Angelo Giovanni Bonadio, MD

Pathological Anatomy 1, University of Pisa, Pisa, Italy 